\title{
Endogenous epitope-tagging of Tet1, Tet2 and Tet3 identifies TET2 as a naïve pluripotency marker
}

\author{
Raphaël Pantier (1), Tülin Tatar, Douglas Colby, Ian Chambers (0)
}

Tet1, Tet2, and Tet3 encode DNA demethylases that play critical roles during stem cell differentiation and reprogramming to pluripotency. Although all three genes are transcribed in pluripotent cells, little is known about the expression of the corresponding proteins. Here, we tagged all the endogenous Tet family alleles using CRISPR/Cas9, and characterised TET protein expression in distinct pluripotent cell culture conditions. Whereas TET1 is abundantly expressed in both naïve and primed pluripotent cells, TET2 expression is restricted to the naïve state. Moreover, TET2 is expressed heterogeneously in embryonic stem cells (ESCs) cultured in serum/leukemia inhibitory factor, with expression correlating with naïve pluripotency markers. FACS-sorting of ESCS carrying a Tet2 $2^{\text {Flag-IRES-EGFP }}$ reporter demonstrated that TET2-negative cells have lost the ability to form undifferentiated ESC colonies. We further show that TET2 binds to the transcription factor NANOG. We hypothesize that TET2 and NANOG co-localise on chromatin to regulate enhancers associated with naïve pluripotency genes.

DOI 10.26508/Isa.201900516 | Received 7 August 2019 | Revised 19 September 2019 | Accepted 19 September 2019 | Published online 3 October 2019

\section{Introduction}

Ten-eleven translocation (TET) family proteins are responsible for active DNA demethylation by sequential oxidation of 5-methylcytosine into 5-hydroxymethylcytosine, 5-formylcytosine, and 5carboxylcytosine $(1,2)$. TET proteins contribute to DNA demethylation in naive embryonic stem cells $(\operatorname{ESCS})(3,4,5,6)$ and their activity is required both for proper differentiation $(7,8)$ and for reprogramming to pluripotency (9). TET proteins are also critical for embryonic development, as Tet1/2/3 triple-knockout embryos cannot proceed beyond gastrulation (10).

Although genetic studies indicate that TET proteins have redundant activities, the low level of sequence conservation outside the catalytic domain suggests that they may also exert distinct functions (11, 12). Indeed, Tet1, Tet2, and Tet3 have different expression patterns during development and in adult tissues (13). TET proteins also interact with partner proteins such as OGT and $\operatorname{Sin} 3 a$ complex members, which might promote functions independent of TET catalytic activity $(14,15,16,17)$.

Because of the lack of reliable commercial antibodies and reporter systems, TET protein expression, particularly at the single cell level, remains poorly characterized. In this study, we used CRISPR/Cas9 in ESCS to tag all endogenous Tet1, Tet2, and Tet3 alleles with antibody epitopes and fluorescent reporters. These cellular reagents allowed the visualisation and the functional analysis of TET proteins in pluripotent cells.

\section{Results}

TET proteins present distinct expression patterns in ESCS

To visualise endogenous TET protein expression in ESCS, we generated knockin alleles using CRISPR/Cas9. Donor templates (targeting vectors or single-stranded oligonucleotides) were used to add epitope tags in frame with the TET protein coding sequences (Figs 1A and fig S1 for a summary of all cell lines). Initially, a targeting vector containing a puromycin resistance cassette $\left(\text { Puro }{ }^{\mathrm{R}} \text { ) was used to add the triple Flag epitope tag (Flag) }\right)_{3}$ at the C-terminus of TET1, resulting in the generation of heterozygous Tet1 ${ }^{\text {Flag-IP/+ }}$ ESC clones (Fig S2). To obtain a cell line expressing only tagged versions of TET1, the remaining wild-type allele of Tet $1^{\text {Flag-IP/+ }}$ clone C10 was re-targeted using a vector with an EGFP reporter to give Tet1 Flag-IP/Flag-IGFP cells (Fig S3). For subsequent modifications of Tet alleles, single-stranded DNA (ssDNA) oligonucleotides were used as donor templates for homologous recombination, as they result in high targeting efficiencies and do not require the use of a selection cassette (18). This alternative strategy was used to fuse a V 5 epitope to the $C$ terminus of TET2 in Tet1 $^{\text {Flag-IP/Flag-IGFP }}$ clone C1 (Fig S4). In clones C2 and C3, both Tet2 alleles were successfully modified in a single step, resulting in the generation of double-tagged Tet1 $1^{\text {Flag-IP/Flag-IGFP; }}$

Tet2 ${ }^{V 5 / V 5}$ ESC clones. To generate an ESC line carrying all six modified Tet alleles, Tet1 ${ }^{\text {Flag-IP/Flag-IGFP. }}$ Tet2 ${ }^{\text {V5/V5 }}$ clone $\mathrm{C} 3$ was modified using a SSDNA that fused a HA tag to the $\mathrm{C}$ terminus of TET3. PCR genotyping identified two Tet7 Flag-IP/Flag-IGFP. $_{\text {Tet2 }}^{\text {V5/V5 }}{ }^{\text {Tet3 }}{ }^{\text {HA/HA }}$ clones (Fig S5), and

UK Medical Research Council Centre for Regenerative Medicine, Institute for Stem Cell Research, School of Biological Sciences, University of Edinburgh, Edinburgh, Scotland

Correspondence: ichambers@ed.ac.uk 
A

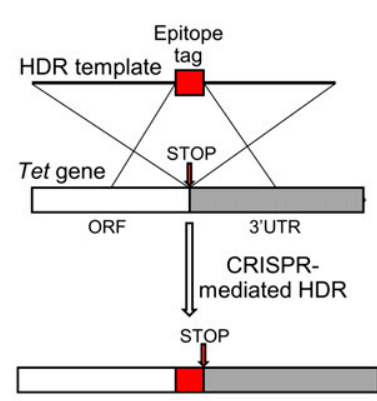

B

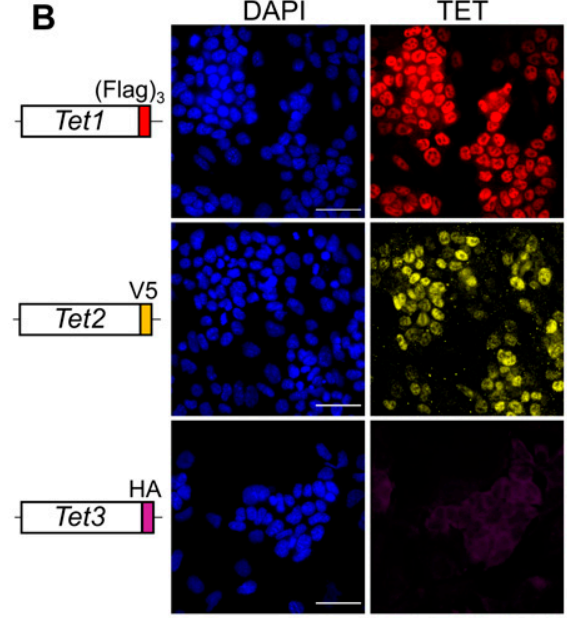

HA antibody positive control

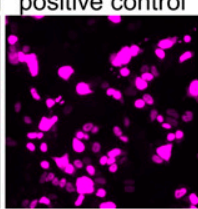

Figure 1. TET protein expression and heterogeneity in ESCS.

(A) General strategy for generating tagged Tet knockin alleles. ESCs were co-transfected with a gRNA designed near the stop codon and a repair template (singlestranded oligo or targeting vector) containing an epitope tag (Flag, V5 or HA). (B, C) Immunofluorescence for Flag (TET1, red), V5 (TET2, yellow), and HA (TET3, magenta) in Tet ${ }^{\text {tag/tag }}$ ESCS cultured in serum/LIF (B) or 2i/LIF (C). (B) Wild-type E14Tg2a ESCs transfected with an HA-NANOG expression plasmid provided a positive control (B). Scale bars: $50 \mu \mathrm{m}$. (D) Immunofluorescence for V5 (yellow) in Tet1 ${ }^{\mathrm{V} / \mathrm{V} 5}$ ESCS (top) and Tet ${ }^{\text {tag } / \text { tag }}$ ESCS (bottom) cultured in serum/ LIF. Samples were imaged and processed under the same conditions to allow a direct comparison of TET1 and TET2 expression levels. Scale bars: $50 \mu \mathrm{m}$.
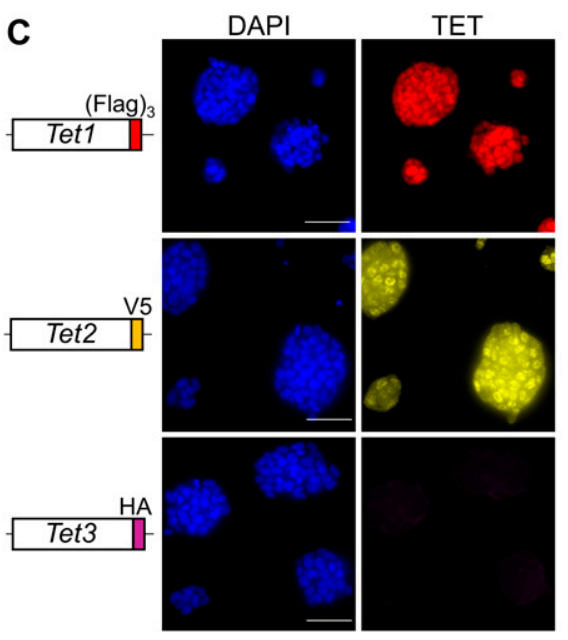

D

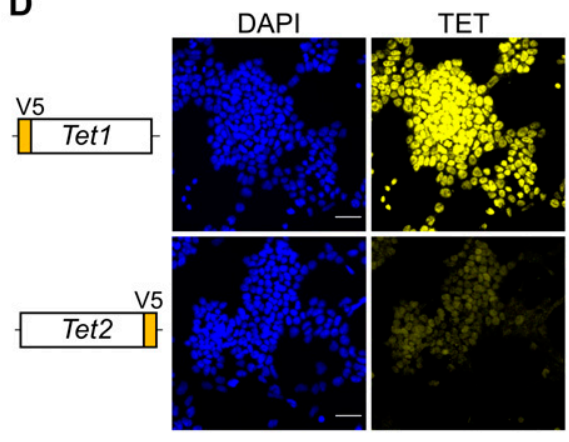

Sanger sequencing confirmed that clone $\mathrm{C} 7$ has both Tet3 alleles appropriately modified, which we refer to as Tet ${ }^{\text {tag/tag }}$ ESCS.

To investigate the expression profile and single cell heterogeneity of TET1, TET2, and TET3 proteins, we performed immunofluorescence analyses on Tet $^{\text {tag/tag }}$ ESCs using antibodies recognising flag (TET1), V5 (TET2), or HA (TET3) epitope tags. In serum/Leukemia inhibitory factor (LIF), TET1 is expressed in most cells at relatively homogenous levels within the population (Fig 1B). In contrast, TET2 is heterogeneously expressed with a "salt and pepper" pattern composed of a mixture of TET2-positive and TET2-negative ESCs (Fig 1B). TET3 was undetectable (Fig 1B). To further explore TET protein expression in the naive state, we cultured Tet ${ }^{\text {tag/tag }}$ ESCS in the presence of LIF and inhibitors of MEK and GSK3 $\beta$ (2i/LIF). In this condition, ESCS form dome-shaped colonies and homogenously express naïve pluripotency markers (19). In 2i/LIF, both TET1 and TET2 present a more homogenous expression pattern (Fig 1C). Once again TET3 protein was undetectable (Fig 1C). These data on TET protein expression are in accord with the relative expression of Tet mRNAs (Fig S6A and B). Interestingly, all Tet mRNAs are expressed at lower levels in 2i/LIF than serum/LIF.
Although the preceding analyses demonstrated that TET family proteins are differentially expressed in ESCS, the use of different epitope tags did not allow a direct comparison of expression levels of different TET proteins. Therefore, to allow the relative quantification of TET1 and TET2 proteins, we targeted Tet1 in E14Tg2a ESCs with an ssDNA to introduce the V5 epitope tag (Fig S7A). Two clones in which both Tet1 alleles were tagged by V5 were obtained (Fig S7B). We next performed comparative analyses of Tet $7^{V 5 / V 5}$ ESCS with Tet $^{\text {tag/tag }}$ ESCs in which an identical V5 epitope tag was fused to TET2. We confirmed that both cell lines retain similar self-renewal efficiencies as wild-type E14Tg2a ESCs (Fig S8A) and express normal levels of the pluripotency factor NANOG (Fig S8B and C). Comparative immunostaining of Tet ${ }^{15 / V 5}$ ESCS with Tet $^{\text {tag/tag }}$ ESCS using a V5 antibody showed that TET1 is expressed at much higher levels than TET2 in serum/LIF (Fig 1D). Consistent with low protein abundance, TET2 was undetectable by Western blot but could be detected after enrichment by immunoprecipitation, showing a band at the predicted size (210 kD) for the full-length TET2-V5 protein (Fig S6C).

Together, these analyses revealed for the first time the relative expression of TET proteins expression at the single cell level in ESCS. 


\section{TET2 marks self-renewing ESCs in serum/LIF}

To further characterise TET2 function, we generated a Tet2 ${ }^{\text {Flag-IRES- }}$ EGFP reporter cell line from E14Tg2a ESCS. After transfection with CRISPR/Cas9 and a targeting vector, EGFP ${ }^{+}$ESCs were sorted into single wells and expanded (Fig S9A). PCR analyses identified nine ESC clones in which (Flag) ${ }_{3}$-IRES-EGFP was targeted to the $3^{\prime}$ end of Tet2 (Fig S9B). Of these, four clones did not produce PCR products of wild-type alleles, indicating that both Tet2 alleles were modified (Fig S9C). Co-immunofluorescence analysis of ESCS cultured in serum/LIF confirmed the heterogeneous TET2 expression pattern and indicated that TET2 (Flag) expressing cells were also fluorescently marked by the cytoplasmic EGFP transcriptional reporter (Fig 2A). FACS of ESCs cultured in serum/LIF allowed the selection of Tet2 ${ }^{\text {Flag-IRES-EGFP }}$ cells based on their TET2 expression level, using the nonfluorescent parental cell line (E14Tg2a) as a negative control (Figs 2B and Fig S10). To investigate the self-renewal efficiency of ESCS expressing distinct TET2 levels, ESCS cultured in serum/LIF were FACS-sorted into TET2-positive and TET2-negative populations. The cells were then plated at clonal density and stained for AP following $7 \mathrm{~d}$ of culture in serum/ LIF condition. Strikingly, TET2-negative ESCS formed almost no self-renewing ESC colonies (Fig 2C). In contrast, TET2-positive ESCS showed a similar number and proportion of AP-stained colonies compared with the bulk-sorted control and parental cell lines (Fig 2C).

To examine transcriptional differences between TET2-positive and TET2-negative ESC populations, quantitative reverse transcription PCR (RT-qPCR) analysis was performed on selected transcripts (Fig 2D). As expected, Tet2 mRNA expression was high in TET2-positive ESCS, and dramatically decreased in TET2negative ESCS. The transcript levels of the pluripotency factor Oct4 were decreased by $50 \%$ in TET2-negative ESCs compared with the bulk-sorted control or the parental cell line. This reduction was more pronounced with the naive markers Nanog and Esrrb.

Together, these data suggest that TET2 expression is tightly associated with naive pluripotency marker expression and efficient ESC self-renewal.
A

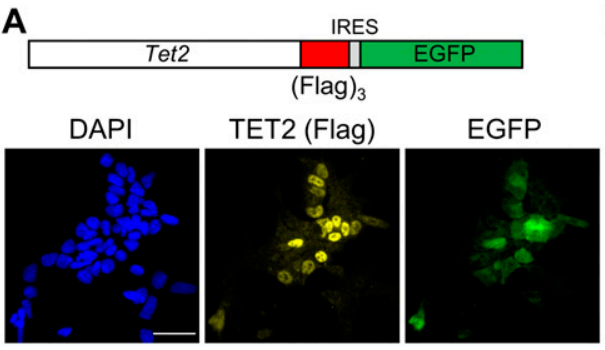

B

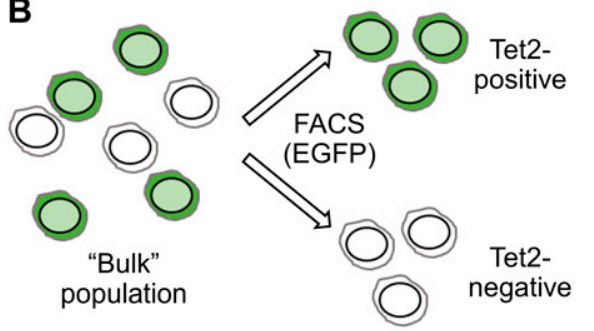

C

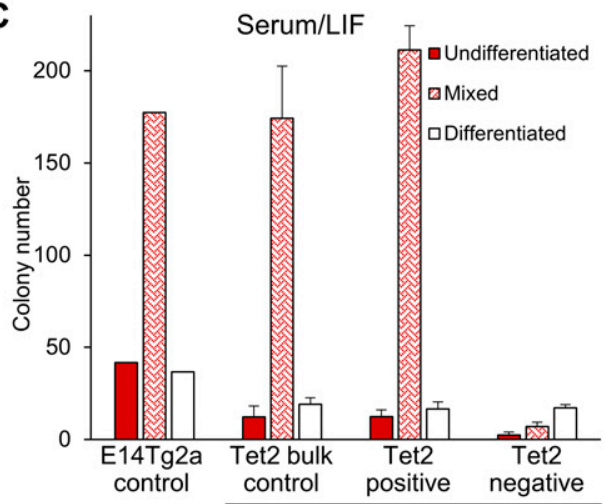

Tet2 ${ }^{\text {Flag-IRES-EGFP ESCS }}$
D
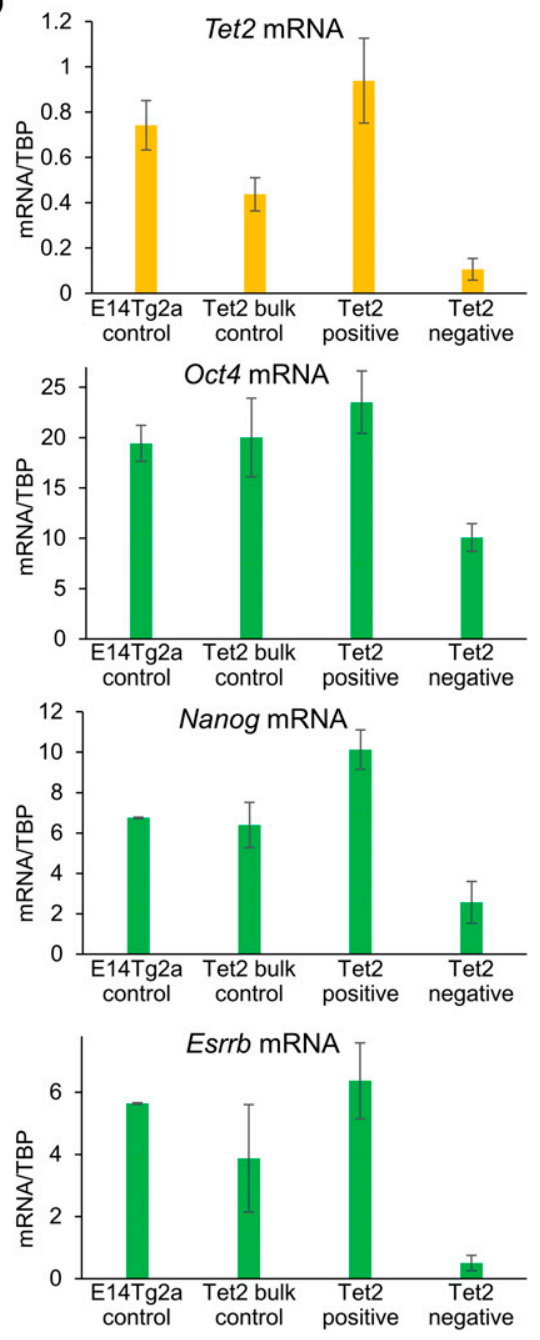

Figure 2. TET2 marks self-renewing ESCs in serum/LIF culture condition.

(A) Co-immunofluorescence for Flag (TET2, yellow) and

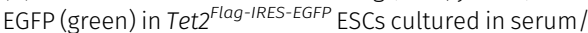
LIF. Scale bar: $50 \mu \mathrm{m}$. (B) General strategy for sorting TET2-EGFP-positive and TET2-EGFP-negative Tet2 ${ }^{\text {Flag }}$ IRES-EGFP ESCS. (C) Clonal self-renewal assays of FACSsorted Tet2 ${ }^{\text {Flag-IRES-EGFP }}$ ESCS (or the wild-type parental cell line). Error bars: SD of the mean $(n=3)$. (D) Quantitative mRNA expression in FACS-sorted

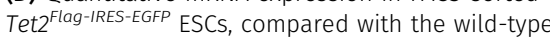
parental cell line. Error bars: SD of the mean $(n=2)$. 


\section{TET2 interacts with NANOG and co-localises at ESC enhancers}

NANOG is heterogeneously expressed in ESCS and its expression level is directly related to self-renewal efficiency $(20,21)$. As both TET2 and NANOG behave as naive pluripotency markers and were reported to interact with each other (22), we further investigated the relationship between these two proteins.

To compare the expression patterns of TET2 and NANOG, we performed co-immunofluorescence in ESCs grown in serum/ LIF (Fig 3A). Quantitation of immunofluorescence in single cells showed that the vast majority of TET2-positive cells co-express NANOG (Fig 3B). This observation further confirms the correlation between TET2 and NANOG that we identified at the mRNA expression level (Fig 2D). However, TET2 marks a larger population of cells than NANOG, resulting in the detection of $\mathrm{TET}^{+} / \mathrm{NANOG}^{-} \mathrm{ESCS}$ (Fig $3 \mathrm{~A}$ and $\mathrm{B}$ ).

To examine the physical interaction between TET2 and NANOG, we performed co-immunoprecipitations in ESCs using differently truncated TET2 constructs (Fig S11A). Interestingly, both TET2 N terminus $(1-1,221)$ and $C$ terminus $(924-1,911)$ interact with NANOG (Fig S11B). Non-overlapping TET2 truncations (1-828 and 924-1,377) retained their interaction with NANOG (Fig S11C). This suggests the presence of at least two NANOG-binding regions within TET2. Interestingly, the stronger interaction with full-length TET2 compared with TET2 $\mathrm{N}$ - and C-terminal fragments (Fig S11C), suggests that NANOG-binding regions act in a cooperative manner.

To further explore the interaction between TET2 and NANOG, we investigated the chromatin-binding profile of these two proteins in ESCS. Previously published TET2 (23) and NANOG (24) ChIP-seq datasets were analysed and compared, with a particular focus on the pluripotency gene regulatory network which controls ESC selfrenewal (25). TET2 and NANOG ChIP-seq signals (and their respective input controls) were visualised as heat maps at ESC enhancers, defined as sites co-bound by OCT4-SOX2-NANOG (24). Both TET2 and NANOG are centrally enriched at ESC enhancers (Fig 3C). To validate this observation, we examined TET2 and NANOG ChIP-seq signal at relevant pluripotency genes (Nanog, Oct4, Esrrb, Klf4, and Prdm14) using a genome browser. Interestingly, TET2- and NANOG-binding profiles are highly similar at these loci with most peaks co-localising within ESC "super-enhancers" (24) (Fig 3D).
A

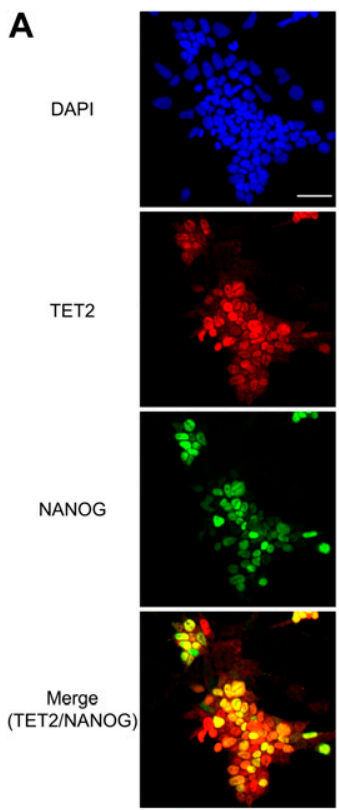

B

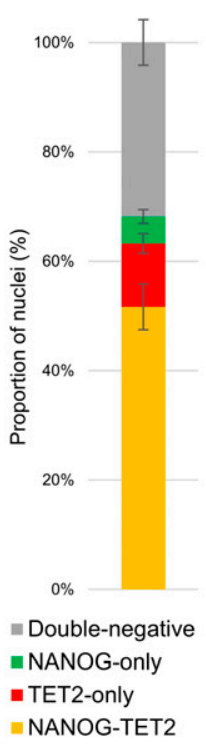

C

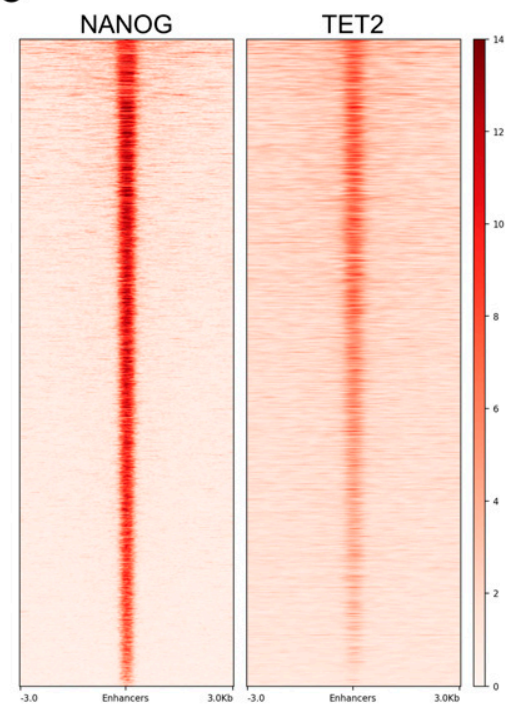

Figure 3. TET2 interacts with NANOG and co-localises at ESC enhancers.

(A) Co-immunofluorescence for Flag (red) and NANOG

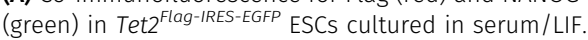
Scale bar: $50 \mu \mathrm{m}$. (B) Quantification of TET2/NANOG co-immunofluorescence (as in panel [A]) in four independent Tet2 ${ }^{\text {Flag-IRES-EGFP }}$ ESC clones cultured in serum/LIF. For each clone, nuclei were counted in two independent fields of view. (C) Heat maps showing NANOG (24) and TET2 (23) RPKM-normalised ChIP-seq signal at ESC enhancers. (D) Genomic snapshots showing TET2 (red) and NANOG (green) ChIP-seq signal at selected pluripotency loci. Blue bars: ESC superenhancers (24).

D
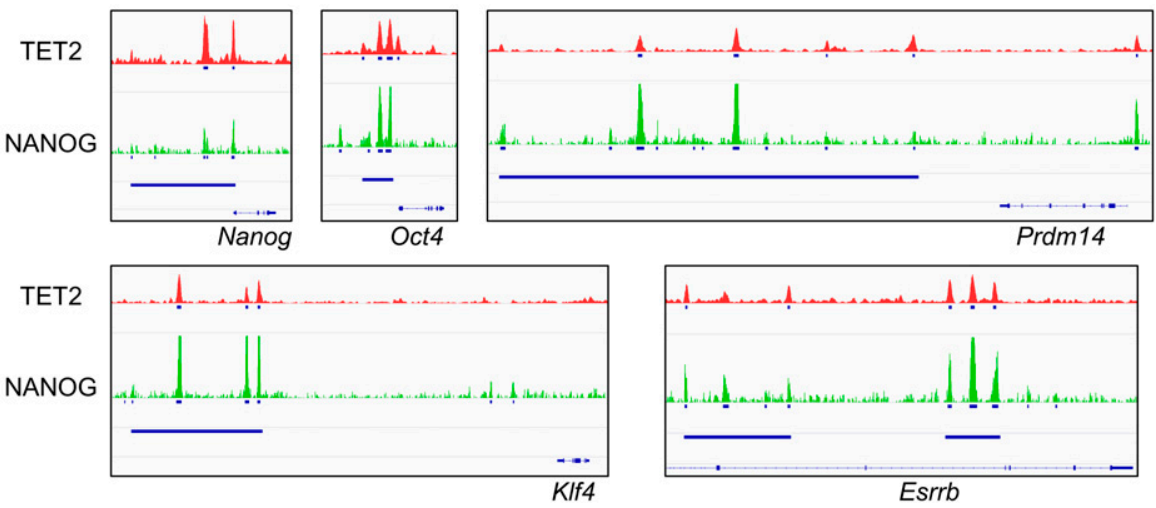
Together, these results indicate that TET2 and NANOG physically interact and co-localise on chromatin to regulate the pluripotency gene regulatory network.

\section{TET proteins are dynamically expressed during the transition from naïve to primed pluripotency}

Recent studies demonstrated that ESCs can be driven from a naive to a primed pluripotent state in vitro, reflecting a change from a pre- to post-implantation epiblast molecular signature. This transition is accompanied by global epigenomic and transcriptional changes (26), to which TET proteins might contribute (27).

To examine the expression of Tet family genes in the primed pluripotent state, we used two different culture systems: Epiblast-like cells (EpiLC) (28) and Epiblast stem cells (EpiSC) (29). First, we examined the mRNA levels of Tet1, Tet2, and Tet3 by RT-qPCR (Fig 4A). In EpiLCs (24 and $48 \mathrm{~h}$ ), Tet1 transcript levels are similar to naive ESCs cultured in 2i/LIF. In contrast, Tet1 mRNA is decreased by threefold to fivefold in EpiSCs compared with ESCs cultured in serum/LIF and 2i/LIF, respectively. Tet2 transcripts are dramatically decreased both in EpiLCs and EpiSCs compared with ESCs, further confirming that Tet2 behaves as a naïve pluripotency marker. In EpiLCs (48 h) and EpiSCs, Tet3 is transcribed at levels which are similar to ESCs cultured in serum/LIF, where TET3 protein is undetectable (Fig 1B)

To extend our analysis to the expression of TET proteins in the primed state, we performed EpiLC and EpiSC differentiation with our Tet $^{\text {tag/tag }}$ ESC line, followed by immunofluorescence for TET1 (Flag), TET2 (V5), or TET3 (HA), together with the control marker OCT6 (26). Both in EpiLCs (Fig 4B) and in EpiSCs (Fig 4C), TET1 was the only detected protein, showing a homogenous expression pattern. However, a long exposure time was required to image TET1 in EpiSCs, indicating a lower expression level than EpiLCs/ESCS and confirming the results from RT-qPCR analysis (Fig 4A).

To further explore changes in TET protein expression during the transition from naive to primed pluripotency, we performed a time course EpiSC differentiation experiment with Tet ${ }^{\text {tag/tag }}$ ESCS. Surprisingly, TET1 protein showed highly dynamic changes (Fig 4D). Up to day 3 of the EpiSC differentiation protocol, TET1 expression was progressively lost in the whole population. On day 4, both TET1 and NANOG were re-expressed at high levels in a subset of cells, which
A
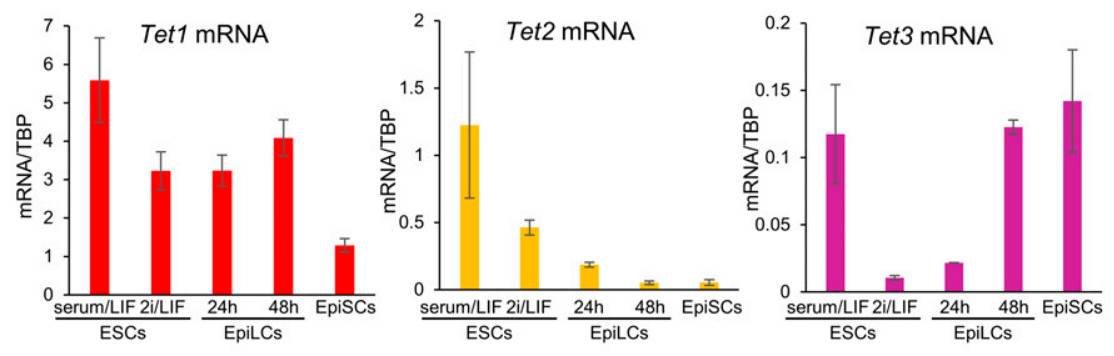

\section{B}

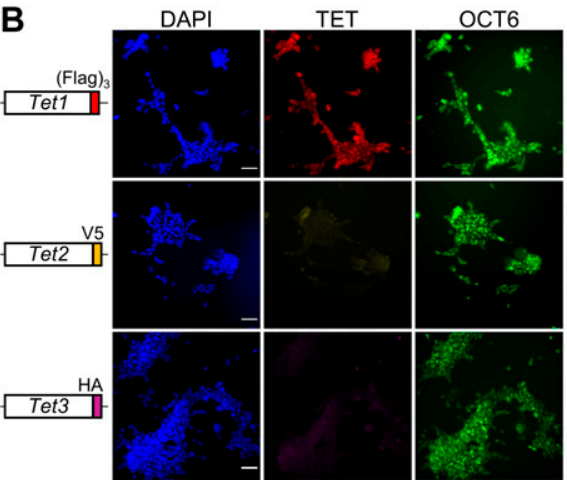

C

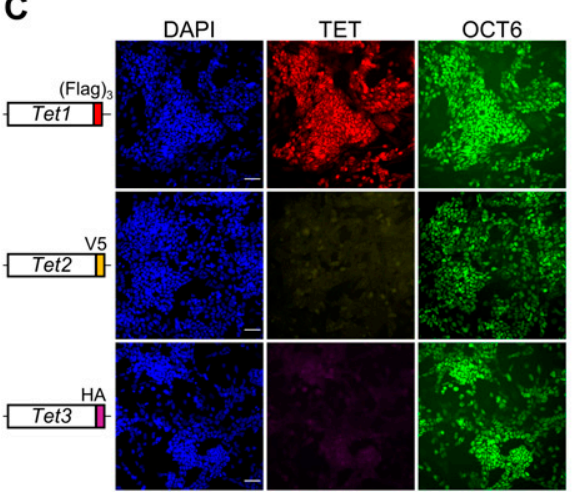

D

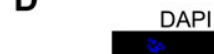

ESC (Serum/LIF)

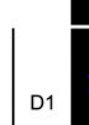

D

DAPI

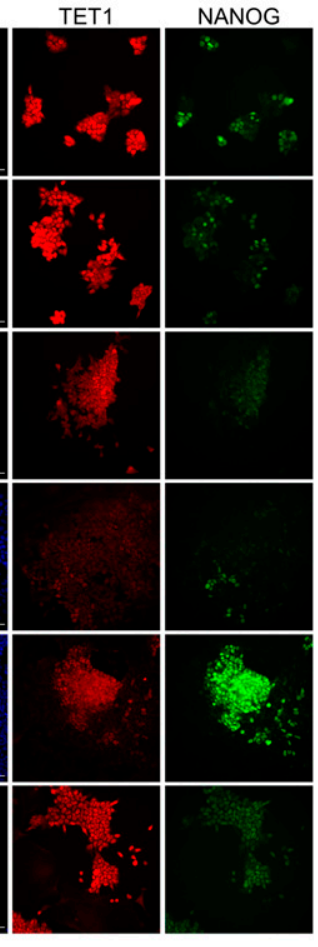

Figure 4. TET protein dynamics during the transition from naive to primed pluripotency.

(A) Quantitative mRNA expression in E14Tg2a ESCS cultured in naïve conditions (serum/LIF or $2 \mathrm{i} / \mathrm{LIF}$ ) and differentiated into primed EpiLCs (24 and $48 \mathrm{~h}$ ) or EpiSCs (passage 15). Error bars: SD of the mean $(n \geq 2)$. (B, C) Co-immunofluorescence for OCT6 (green) and Flag (TET1, red) or V5 (TET2, yellow) or HA (TET3, magenta) in Tet ${ }^{\text {tag/tag }}$ EpiLCs (48 h) (B) or in Tet ${ }^{\text {tag/tag }}$ EpisCs (passage 11) (C) cultured in activin/FGF. Scale bars: $50 \mu \mathrm{m}$. (D) Time course coimmunofluorescence for Flag (TET1, red) and NANOG (green) in Tet ${ }^{\text {tag/tag }}$ cells during EpiSC differentiation (arrow) from serum/LIF culture condition. Samples were imaged and processed under the same conditions to allow a direct comparison of TET1 and NANOG expression levels between different time points. Scale bars: $50 \mu \mathrm{m}$. 
may mark future EpiSCS, as TET1 and NANOG expression was homogenous by passage 3 (at lower levels than day 4). Interestingly, TET2 followed a similar expression dynamics during early EpiSC differentiation (Fig S12A), although its expression was completely lost in stable EpiSCs (Fig $4 \mathrm{C}$ ). In contrast, TET3 remained undetectable throughout EpiSC differentiation (Fig S12B). A similar time course immunofluorescence experiment was performed in Tet $^{\text {tag/tag }}$ cells during EpiLC differentiation (from 24 to $72 \mathrm{~h}$ ). This analysis did not reveal any change in TET1 protein expression compared with 2i/ LIF (Fig S13A), whereas TET2 and TET3 could not be detected at any time point (Fig S13B and C), in agreement with their low transcription levels (Fig 4A).

Together, these experiments revealed dynamic changes in TET protein expression during the transition from naïve to primed pluripotency.

\section{Discussion}

In this study, we comparatively assessed the expression of all Tet family genes in pluripotent cells. Immunofluorescence analyses revealed that TET1 protein is expressed in both naive and primed pluripotent cells, whereas TET2 is exclusively expressed in naive ESCS. Furthermore, TET1 is homogeneously expressed in serum/LIF, whereas TET2 is expressed only in a subset of cells. This differential expression suggests that TET1 and TET2 might exert distinct functions in pluripotent cells, which is supported by recent studies. Knockdown experiments indicated that TET1 and TET2 control the methylation of distinct genomic regions in ESCS (11). In addition, the ectopic expression of TET2 (but not TET1) reprograms cells from primed to naïve pluripotency (12).

Our work indicated that TET2 is a naive pluripotency marker. In serum/LIF, TET2 specifically marks self-renewing ESCS and is associated with the expression of other heterogeneously expressed factors controlling the naïve state like NANOG (21) and Esrrb (30). Conversely, TET2-negative cells present low levels of naive pluripotency markers and are unable to form AP-positive colonies. In addition, TET2 expression becomes homogenous during the transition from serum/LIF to $2 \mathrm{i} / \mathrm{LIF}$, which maintains ESCS in a naive state. Conversely, TET2 expression is abolished during the transition to the primed state, either with the EpiLC or the EpiSC differentiation protocol. Further molecular characterisation on TET2-positive and TET2negative ESCS will be required to reveal genome-wide transcriptional and methylation changes between these two cell populations.

We demonstrated that TET2 and NANOG are mostly co-expressed in ESCS cultured in serum/LIF. This observation could be extended to the analysis of TET2 expression in the inner cell mass of the preimplantation blastocyst, where NANOG presents a "salt and pepper" expression pattern, which is critical to determine the balance between epiblast and primitive endoderm (31). Furthermore, NANOG transiently fluctuates between high and low expression states in ESCS (21). As TET2 is a direct transcriptional target of NANOG (32), its expression might fluctuate in a similar manner, explaining the correlation between TET2 and NANOG expression in ESCS.

In this study, we have shown that TET2 physically interacts with NANOG and co-localises at pluripotency enhancers. As TET2 lacks a
DNA-binding domain, it might be targeted by NANOG to these loci to maintain them in a demethylated state. A recent study reported that TET2 could be efficiently ChIPed only after extensive cross-linking with formaldehyde plus disuccinimidyl glutarate (33), suggesting that TET2 interacts indirectly with chromatin. TET2 localises to completely different loci in ESCS and hematopoietic cells (33), supporting a model in which TET2 is targeted to chromatin via protein-protein interactions in a context-dependent manner. In contrast, TET1 has a CXXC domain, which recruits this protein mostly to CpG islands (17, 34). Supporting this model, Tet2 knockout causes hypermethylation of enhancers in ESCS, whereas Tet1 knockout has no effect on the methylation status of these genomic regions (35). More recently, TET2 was shown to promote enhancer demethylation by interacting with $\mathrm{C} / \mathrm{EBP} \alpha, \mathrm{Klf} 4$ and Tfcp2l1 at distinct stages of induced pluripotent stem cell reprogramming (36).

Here, time course experiments allowed the visualisation of TET expression changes during the transition from naive to primed pluripotency. Interestingly, TET1/2 and NANOG showed similar dynamics during early EpiSC differentiation. The global and transient decrease in NANOG/TET expression might mark the recently described "formative" pluripotent state (37). However, TET proteins did not show dynamic expression changes during EpiLC differentiation, which might be explained by a rapid and homogenous transition to the primed state (28) without passing through a "formative" pluripotent state. In addition, TET1 is expressed at higher levels in EpiLCs compared with EpiSCs. These observations highlight differences between the EpiLC and EpiSC differentiation protocols.

In conclusion, we generated knockin alleles of Tet family genes with epitope tags or fluorescent reporters, providing a robust characterisation of TET protein expression dynamics and single-cell heterogeneity in pluripotent cells. The engineered ESC lines produced in this study could be further exploited to study TET1/2/3 protein expression in vivo in transgenic mouse models.

\section{Materials and Methods}

\section{Cell culture}

All the cell lines in this study were derived from E14Tg2a (38) and incubated in a $37^{\circ} \mathrm{C} / 7 \% \mathrm{CO}_{2}$ incubator. ESCs were cultured on gelatincoated plates. Composition of the serum/LIF medium: Glasgow Minimum Essential Medium (Cat. no. G5154; Sigma-Aldrich), 10\% fetal bovine serum, 1× L-glutamine (Cat. no. 25030-024; Invitrogen), 1× pyruvate solution (Cat. no. 11360-039; Invitrogen), 1× MEM nonessential amino acids (Cat. no. 11140-036; Invitrogen), 0.1 mM 2-mercaptoethanol (ref. 31350010; Gibco), and $100 \mathrm{U} / \mathrm{ml}$ LIF (made in-house).

For 2i/LIF ESC culture (19), serum-free N2B27 medium was prepared: 1:1 vol/vol mix of DMEM:F12 (Cat. no. 12634010; Gibco) and Neurobasal (Cat. no. 21103049; Gibco), 1× L-glutamine (Cat. no. 25030-024; Invitrogen), 1× MEM nonessential amino acids (Cat. no. 11140-036; Invitrogen), 0.1 mM 2-mercaptoethanol (ref. 31350010; Gibco), 1× N2 supplement (Cat. no. 17502048; Gibco), 1× B27 supplement (Cat. no. 17504044; Gibco). 1 MM PD0325901 (Cat. no. 1408; Axon), 3 MM CHIR99021 (Cat. no. 1386; Axon), and $100 \mathrm{U} / \mathrm{ml}$ LIF were added freshly to the medium. 
EpiSC lines were derived in vitro from ESCS (29). $3 \times 10^{4}$ ESCS were plated in a well of a six-wells plate with serum/LIF medium (see composition above). After $24 \mathrm{~h}$, the medium was switched to N2B27 medium (see composition above) supplemented with $20 \mathrm{ng} / \mathrm{ml}$ human activin A (Cat. no. 120-14E; PeproTech) and $10 \mathrm{ng} / \mathrm{ml}$ human Fgf basic (Cat. no. 233-FB-025/CF; R\&D Systems). The cells were submitted to daily media changes and passaged at day 5 of the protocol in six-well plates coated with $7.5 \mu \mathrm{g} / \mathrm{ml}$ bovine fibronectin. The cells were maintained in N2B27 medium supplemented with Activin/Fgf and passaged every 2-3 d. Homogenous EpisCs were derived within 10 passages.

EpiLC differentiation was performed as described in (39). ESCS were adapted to 2i / LIF culture for at least three passages on poly-Lornithine (Cat. no. P3655; Sigma-Aldrich) and laminin-coated wells (Cat. no. 354232; BD Biosciences) of a six-well plate. $2.5 \times 10^{5}$ ESCS were plated on a well of six-well plate pretreated with $16.6 \mu \mathrm{l} / \mathrm{ml}$ fibronectin (Cat. no. FC010; Millipore) and containing EpiLC medium: N2B27 medium (see the composition above) supplemented with 20 $\mathrm{ng} / \mathrm{ml}$ human activin A (Cat. no. 120-14E; PeproTech), $12 \mathrm{ng} / \mathrm{ml}$ human Fgf basic (Cat. no. 233-FB-025/CF; R\&D Systems) and 1\% knockout serum replacement (Cat. no. 10828-028; Gibco). The cells were submitted to daily media changes and collected for analyses.

\section{Self-renewal assays}

Cells were collected by trypsinisation and resuspended in PBS (Cat. no. D8537; Sigma-Aldrich) supplemented with $2 \%$ fetal bovine serum at a concentration of around $1 \times 10^{6}$ cells $/ \mathrm{ml}$. The cell suspension was passed through a cell strainer and kept on ice until cell sorting (FACSAria II; Becton Dickinson). Single cells were gated using the forward-scattered light and side-scattered light parameters. Autofluorescent (dead) cells were also discarded. Gates for selecting EGFP fluorescent cells ("GFP B 525/50") were drawn using the nonfluorescent parental cell line (E14Tg2a ESCS) as a negative control. Tet2 bulk and E14Tg2a WT control cells were processed by FACS with no selection based on EGFP fluorescence. 600 cells were directly sorted in gelatin-coated wells of six-well plates containing serum/ LIF medium (see the composition above). After $7 \mathrm{~d}$ of culture, the cells were washed in PBS and incubated for $1 \mathrm{~min}$ in a fixative solution made by mixing $25 \mathrm{ml}$ of citrate solution (18 mM citric acid, $9 \mathrm{mM}$ sodium citrate, and $12 \mathrm{mM} \mathrm{NaCl}$ ), $8 \mathrm{ml}$ of formaldehyde solution (37\% $\mathrm{vol} / \mathrm{vol}$ in water), and $65 \mathrm{ml}$ of acetone. Fixed cells were washed in distilled water and stained for AP expression using a leukocyte AP kit (Cat. no. 86R-1KT; Sigma-Aldrich). Colonies were counted and categorised according to their morphology and AP staining.

\section{CRISPR-mediated homologous recombination}

To modify endogenous Tet1/2/3 alleles, a double-strand break was generated at desired genomic loci using Cas9 and a synthetic gRNA (18). gRNAs were designed (http://crispr.mit.edu/) and cloned into Cas9/gRNA co-expression plasmids (pX330; Addgene, or derivatives).

To prevent cutting of targeted alleles by CRISPR/Cas9, donor templates were designed so that the gRNA site is disrupted after homologous recombination. Alternatively, a silent mutation was added in the donor template to disrupt the gRNA PAM sequence
(NGG). Two types of donor templates were used for homologous recombination: targeting vector or ssDNA oligonucleotide. Targeting vectors were cloned by Gibson assembly into a pBluescript backbone and contained a selection cassette (fluorescent reporter or puromycin resistance). The $5^{\prime}$ - and $-3^{\prime}$ homology arms (typical size around $1.5 \mathrm{~Kb}$ ) were amplified by PCR from ESC genomic DNA. SsDNAs (presenting around 60-bp homology arms) were ordered from Integrated DNA Technologies as Ultramer DNA oligonucleotides.

$1 \times 10^{6}$ ESCs were transfected using Lipofectamine 3000 (cat. L3000008; Thermo Fisher Scientific), following the manufacturer's instructions, with both the Cas9/gRNA plasmid and the donor template (targeting vector or SSDNA). After $48 \mathrm{~h}$, ESCs were selected either by FACS sorting (targeting vector with fluorescent reporter or fluorescent Cas9) or by the addition of $0.75 \mu \mathrm{g} / \mathrm{ml}$ puromycin (targeting vector with puromycin resistance cassette). ESC clones were expanded in 24-well plates and genomic DNA was extracted (Cat. no. 69506; QIAGEN) for genotyping Tet alleles. PCR genotyping was performed using forward and/or reverse primers binding outside the homology arms of the donor template, therefore confirming the modification of the endogenous locus. PCR products were submitted to Sanger sequencing to confirm that the desired modification was added in frame with the Tet coding sequence. Correctly targeted ESC clones were expanded, and frozen aliquots were transferred to liquid nitrogen tanks for long term storage.

List of gRNAs used for tagging Tet alleles

\begin{tabular}{l|l} 
Target locus & gRNA site (not including PAM sequence) \\
\hline Tet1 start codon & TTGGAAGGCTTTGCGGGGC \\
\hline Tet1 stop codon & TGCGGGACCCTACAATCGTT \\
\hline Tet2 stop codon & ACAACACATTTGTATGACGC \\
\hline Tet3 stop codon & AGCCGCTGGATCTAGGTGCC \\
\hline
\end{tabular}

List of genotyping primers

\begin{tabular}{ll}
\hline Target locus & Primer sequence \\
\hline Tet1 3' FW1 & CTGATGTATCCCCCGAAGCC \\
\hline Tet1 3' FW2 & CCACGTCCTGCCACTATACC \\
\hline Tet1 3' RV1 & TCGGAGTTGAAATGGGCGAA \\
\hline Tet1 3' RV2 & GGGCTTCTTGTGGCATCTCT \\
\hline Puro FW & GCCGCGCAGCAACAGATGGAA \\
\hline Puro RV & ACCCACACCTTGCCGATGTC \\
\hline EGFP RV & AACTTCAGGGTCAGCTTGCC \\
\hline Tet2 3' FW & ACAGGGTCTGTGACTACGGA \\
\hline Tet2 3' RV1 & ACAGATGCTGTGACCTGTCC \\
\hline Tet2 3' RV2 & CTGTGTCCCACGGTACACA \\
\hline Tet3 3' FW & CCGTGTCCTCTTACGCCTAC \\
\hline Tet3 3' RV & CATGAGGGCAAAAGCACCAC \\
\hline Tet1 5' FW & ACTCCGATGATCCTGCCTCT \\
\hline Tet1 5' RV & TCGGGGTTTTGTCTTCCGTT \\
\hline
\end{tabular}




\section{Immunofluorescence analysis}

Cells were washed with PBS and fixed with 4\% PFA for $10 \mathrm{~min}$ at room temperature. After fixation, the cells were washed with PBS and permeabilised with a solution of PBS containing $0.3 \%$ (vol/ vol) Triton X-100 for 10 min at room temperature. Samples were blocked in blocking buffer (PBS supplemented with $0.1 \%$ [vol/vol] Triton X-100, 1\% [wt/vol] BSA, and 3\% [vol/vol] serum of the same species as the secondary antibodies were raised in) for $1 \mathrm{~h}$ at room temperature. After blocking, the samples were incubated with primary antibodies diluted in blocking buffer overnight at $4^{\circ} \mathrm{C}$. After four washes with PBS containing $0.1 \%$ (vol/vol) Triton X-100, the samples were incubated with fluorescently labelled secondary antibodies diluted in blocking buffer for $1 \mathrm{~h}$ at room temperature in the dark. The cells were washed four times with PBS containing $0.1 \%$ (vol/vol) Triton X-100. DNA was stained with DAPI for $5 \mathrm{~min}$ at room temperature. The cells were washed with PBS for $5 \mathrm{~min}$. The samples were imaged by fluorescence microscopy (Ti-E; Nikon). Images were analysed and processed using the software Fiji.

\section{Immunoprecipitation}

E14/T ESCS were used for producing proteins for immunoprecipitation, as they can replicate and propagate PPyCAG plasmids which carry a polyoma origin of replication (20). $3 \times 10^{6}$ E14/T ESCS were transfected using Lipofectamine 3000 (Cat. no. L3000008; Thermo Fisher Scientific), following the manufacturer's instructions, with $6 \mu \mathrm{g}$ of PPYCAG plasmids carrying a construct of interest.

E14/T ESCs were harvested $24 \mathrm{~h}$ after transfection. The cells were trypsinised, pelleted $\left(5 \mathrm{~min}, 393 \mathrm{~g}, 4^{\circ} \mathrm{C}\right.$ ), and washed twice with cold PBS before lysis in a swelling buffer ( $5 \mathrm{mM}$ Pipes, $\mathrm{pH} 8$, and $85 \mathrm{mM} \mathrm{KCl}$ ) freshly supplemented with $1 \times$ protease inhibitor cocktail (Cat. no. 04693116 001; Roche) and 0.5\% NP-40. After 20 min on ice with occasional shaking, nuclei were pelleted (10 min, $524 \mathrm{~g}, 4^{\circ} \mathrm{C}$ ) and resuspended in $1 \mathrm{ml}$ of lysis buffer (20 mM Hepes, $\mathrm{pH}$ 7.6, $350 \mathrm{mM} \mathrm{KCl}, 0.2 \mathrm{mM}$ EDTA, pH 8, $1.5 \mathrm{mM} \mathrm{MgCl}_{2}$, and $20 \%$ glycerol) freshly supplemented with $0.2 \% \mathrm{NP}-40,0.5 \mathrm{mM}$ DTT, and 1X protease inhibitor cocktail (Cat. no. 04693116 001; Roche). The material was transferred into nonstick microtubes (Cat. no. LW2410AS; Alpha Laboratories) and supplemented with $150 \mathrm{U} / \mathrm{ml}$ of Benzonase Nuclease (Cat. no. 71206; Novagen). The samples were incubated on a rotating wheel for $30 \mathrm{~min}$ at $4^{\circ} \mathrm{C}$. Tubes were centrifuged $\left(16,100 \mathrm{~g}, 30 \mathrm{~min}, 4^{\circ} \mathrm{C}\right)$ and nuclear extracts were collected in clean nonstick tubes. 30-50 $\mu \mathrm{l}$ of nuclear protein extract was boiled in Laemmli buffer as input material.

For immunoprecipitations, $5 \mu \mathrm{g}$ of $\mathrm{V} 5$ antibody (Cat. no. 146796-80; eBioscience) or Flag antibody (Cat. no. F3165; SigmaAldrich) was added to nuclear extracts. For negative controls, $5 \mu \mathrm{g}$ of normal mouse IgG was added to nuclear extracts. Samples were incubated overnight at $4^{\circ} \mathrm{C}$ on a rotating wheel. $30 \mu \mathrm{l}$ of Protein G Sepharose beads (Cat. no. 17061801; GE Healthcare), previously blocked with $0.5 \mathrm{mg} / \mathrm{ml}$ chicken egg albumin, were added to nuclear extracts, followed by a 2 -h incubation at $4^{\circ} \mathrm{C}$ on a rotating wheel. The samples were washed five times in a lysis buffer (20 mM Hepes, pH 7.6, 350 mM KCl, 0.2 mM EDTA, pH 8, 1.5 $\mathrm{mM} \mathrm{MgCl}$, and $20 \%$ glycerol) freshly supplemented with $0.5 \% \mathrm{NP}$ 40 and $0.5 \mathrm{mM}$ DTT. Between each wash, the samples were centrifuged $\left(400 \mathrm{~g}, 1 \mathrm{~min}, 4^{\circ} \mathrm{C}\right)$. After the final wash, beads were boiled in Laemmli buffer to solubilise the immunoprecipitated material.

Protein samples were loaded into 10\% Bis-Tris Gels (Cat. no. NW00102BOX; Novex) with 1X MOPS SDS running buffer (Cat. no. B0001; Novex). $10 \mu$ l of SeeBlue Plus2 prestained protein standard (Cat. no. LC5925; Invitrogen) was used to visualise the protein molecular weight. The electrophoresis was performed at $160 \mathrm{~V}$ for $1 \mathrm{~h}$. Proteins were transferred (overnight at $4^{\circ} \mathrm{C}$ ) to a nitrocellulose membrane at $150 \mathrm{~mA}$ constant current in the presence of a transfer buffer containing $25 \mathrm{mM}$ Tris, $0.21 \mathrm{M}$ glycine, and 10\% methanol. The membrane was blocked for $1 \mathrm{~h}$ at room temperature with $10 \%$ non-fat skimmed milk dissolved in PBS supplemented with $0.1 \%$ Tween. Then, the membrane was incubated for 1 $\mathrm{h}$ at room temperature with primary antibodies diluted to the working concentration (see table) in $5 \%$ nonfat skimmed milk dissolved in PBS supplemented with $0.1 \%$ Tween. The membrane was washed three times with PBS supplemented with $0.1 \%$ Tween, and incubated for $2 \mathrm{~h}$ at room temperature with LI-COR IRDyeconjugated secondary antibodies diluted 1:5,000 in 5\% nonfat skimmed milk dissolved in PBS supplemented with $0.1 \%$ Tween. The membrane was finally washed three times with PBS supplemented with $0.1 \%$ Tween before analysis with the LI-COR Odyssey FC imaging system.

\section{Antibodies}

\begin{tabular}{lll} 
Antibody & Reference & Working dilution (application) \\
\hline Flag & Cat. no. F1804; Sigma-Aldrich & $1: 500$ (immunofluorescence) \\
\hline Flag & Cat. no. F3165; Sigma-Aldrich & $1: 5,000$ (Western blot) \\
\hline HA & Cat. no. Sc-805; Santa Cruz & $1: 50$ (immunofluorescence) \\
\hline V5 & Cat. no. 14-6796-80; eBioscience & $1: 250$ (immunofluorescence), 1:1,000 (Western-blot) \\
\hline NANOG & Cat. no. 14-5761-80; eBioscience & $1: 500$ (immunofluorescence) \\
\hline NANOG & Cat. no. A300-397A; Bethyl Laboratories & $1: 2,000$ (Western-blot) \\
\hline Oct6 & Cat. no. Sc-11661; Santa Cruz & $1: 200$ (immunofluorescence) \\
\hline EGFP & Cat. no. Ab13970; Abcam & $1: 200$ (immunofluorescence) \\
\hline
\end{tabular}




\section{RT-qPCR analysis}

Total RNA was isolated using the RNeasy Plus Mini Kit (cat. 74136; QIAGEN), following the manufacturer's instructions. The quantity and purity of RNA samples were determined using a microvolume spectrophotometer (ND-1000; NanoDrop). RNA was reverse transcribed with SuperScript III (Cat. no. 18080044; Invitrogen) using random hexamer oligonucleotides, following the manufacturer's instructions. Triplicate qPCR reactions were set up with the Takyon SYBR MasterMix (Cat. no. UF-NSMT-B0701; Eurogentec) and analysed using the Roche LightCycler 480 machine. For all qPCR primer pairs, standard curves were performed to assess the amplification efficiency and melting curves were generated to verify the production of single DNA species.

\section{List of qPCR primers}

\begin{tabular}{lll}
$\begin{array}{l}\text { Primer } \\
\text { pairs }\end{array}$ & Forward primer & Reverse primer \\
\hline Esrrb & CGATTCATGAAATGCCTCAA & CCTCCTCGAACTCGGTCA \\
\hline NANOG & AGGATGAAGTGCAAGCGGTG & TGCTGAGCCCTTCTGAATCAG \\
\hline Oct4 & GTTGGAGAAGGTGGAACCAA & CTCCTTCTGCAGGGCTTTC \\
\hline TBP & GGGGAGCTGTGATGTGAAGT & CCAGGAAATAATTCTGGCTCA \\
\hline Tet1 & TTGAGAAGATAGTGTTCACGG & CACTTCTTCTGATCACCCAC \\
\hline Tet2 & CTCATGGAAGAAAGGTATGGAG & GCTCTTGCCTTCTTTACCAG \\
\hline Tet3 & ACTGTCAAGACAGGCTCAG & ATCTCCATGGTACACTGGC \\
\hline
\end{tabular}

\section{ChIP-seq analysis}

ChIP-seq datasets were analysed using the Galaxy platform: https:// usegalaxy.org/ (40). The bioinformatics workflow is available at the following address: https://usegalaxy.org/u/raf4579/w/workflow-chip-seq-1. Raw sequencing data (FASTQ files) were downloaded from the NCBI's Gene Expression Omnibus database. Quality control was performed using the software "FastQC" (41). Samples were filtered to remove contaminating adapter sequences and low-quality reads (cutoff quality score $>20.0$ ). Reads were mapped to the mouse $\mathrm{mm} 9$ reference genome using "Bowtie2" (BAM file output) (42). Reads were mapped only to a unique genomic location $(k=1)$. ChIP-seq peaks were called using the software "MACS2" (BED file output) (43). The immunoprecipitated sample was compared with the genomic input for identifying statistically significant binding sites (q value 0.05). To visualise ChIP-seq datasets on a genome browser, mapped reads (BAM files) were converted into bigwig files using "deepTools" (44). Data were normalised in "reads per kilobase million" (RPKM) to allow the comparison between ChIP-seq datasets. Genomic snapshots were taken using the genome viewer "IGV" (45). To visualise ChIP-seq datasets as heat maps, the software "deepTools" was used (44). RPKM-normalised bigwig files were aligned to ESC enhancers (24).

\section{Data Availability}

Previously published NANOG (24) and TET2 (23) high-throughput sequencing data were obtained from the NCBI's Gene Expression
Omnibus database: NANOG ChIP-seq (GSE44286), TET2 ChIP-seq (GSE57700).

\section{Supplementary Information}

Supplementary Information is available at https://doi.org/10.26508/lsa. 201900516.

\section{Acknowledgements}

We thank Bertrand Vernay for microscopy support. We thank Claire Cryer and Fiona Rossi for assistance with flow cytometry. We are grateful to Kristian Helin (University of Copenhagen) for sharing Tet2 expression plasmids. This work was funded by a UK Medical Research Council grant MR/L018497/1 to I Chambers. R Pantier was supported by a UK Medical Research Council PhD Fellowship. T Tatar was supported by a Darwin Trust of Edinburgh PhD Fellowship.

\section{Author Contributions}

R Pantier: conceptualization, data curation, formal analysis, validation, investigation, visualization, methodology, and writing-original draft, review, and editing.

T Tatar: investigation and methodology.

D Colby: investigation.

I Chambers: conceptualization, resources, supervision, funding acquisition, investigation, project administration, and writing-original draft, review, and editing.

\section{Conflict of Interest Statement}

The authors declare that they have no conflict of interest.

\section{References}

1. Tahiliani M, Koh KP, Shen Y, Pastor WA, Bandukwala H, Brudno Y, Agarwal S, Iyer LM, Liu DR, Aravind L, et al (2009) Conversion of 5-methylcytosine to 5-hydroxymethylcytosine in mammalian DNA by MLL partner TET1. Science 324: 930-935. doi:10.1126/science.1170116

2. Ito S, Shen L, Dai Q, Wu SC, Collins LB, Swenberg JA, He C, Zhang Y (2011) Tet proteins can convert 5-methylcytosine to 5-formylcytosine and 5carboxylcytosine. Science 333: 1300-1303. doi:10.1126/science.1210597

3. Habibi E, Brinkman AB, Arand J, Kroeze LI, Kerstens HHD, Matarese F, Lepikhov K, Gut M, Brun-Heath I, Hubner NC, et al (2013) Whole-genome bisulfite sequencing of two distinct interconvertible DNA methylomes of mouse embryonic stem cells. Cell Stem Cell 13: 360-369. doi:10.1016/ j.stem.2013.06.002

4. Ficz G, Hore TA, Santos F, Lee HJ, Dean W, Arand J, Krueger F, Oxley D, Paul Y-L, Walter J, et al (2013) FGF signaling inhibition in ESCs drives rapid genome-wide demethylation to the epigenetic ground state of pluripotency. Cell Stem Cell 13: 351-359. doi:10.1016/j.stem.2013.06.004

5. Hackett JA, Dietmann S, Murakami K, Down TA, Leitch HG, Surani MA (2013) Synergistic mechanisms of DNA demethylation during transition to ground-state pluripotency. Stem Cell Reports 1: 518-531. doi:10.1016/ j.stemcr.2013.11.010 
6. von Meyenn F, Iurlaro M, Habibi E, Liu NQ, Salehzadeh-Yazdi A, Santos F, Petrini E, Milagre I, Yu M, Xie Z, et al (2016) Impairment of DNA methylation maintenance is the main cause of global demethylation in naive embryonic stem cells. Mol Cell 62: 848-861. doi:10.1016/ j.molcel.2016.04.025

7. Dawlaty MM, Breiling A, Le T, Barrasa MI, Raddatz G, Gao Q, Powell BE, Cheng AW, Faull KF, Lyko F, et al (2014) Loss of Tet enzymes compromises proper differentiation of embryonic stem cells. Dev Cell 29: 102-111. doi:10.1016/j.devcel.2014.03.003

8. Verma N, Pan H, Doré LC, Shukla A, Li QV, Pelham-Webb B, Teijeiro V, González F, Krivtsov A, Chang C-J, et al (2018) TET proteins safeguard bivalent promoters from de novo methylation in human embryonic stem cells. Nat Genet 50: 83-95. doi:10.1038/s41588-017-0002-y

9. Hu X, Zhang L, Mao S-Q, Li Z, Chen J, Zhang R-R, Wu H-P, Gao J, Guo F, Liu W, et al (2014) Tet and TDG mediate DNA demethylation essential for mesenchymal-to-epithelial transition in somatic cell reprogramming. Cell Stem Cell 14: 512-522. doi:10.1016/j.stem.2014.01.001

10. Dai H-Q, Wang B-A, Yang L, Chen J-J, Zhu G-C, Sun M-L, Ge H, Wang R, Chapman DL, Tang F, et al (2016) TET-mediated DNA demethylation controls gastrulation by regulating Lefty-Nodal signalling. Nature 538: 528-532. doi:10.1038/nature20095

11. Huang Y, Chavez L, Chang X, Wang X, Pastor WA, Kang J, Zepeda-Martínez JA, Pape UJ, Jacobsen SE, Peters B, et al (2014) Distinct roles of the methylcytosine oxidases Tet1 and Tet2 in mouse embryonic stem cells. Proc Natl Acad Sci U S A 111: 1361-1366. doi:10.1073/pnas.1322921111

12. Fidalgo M, Huang X, Guallar D, Sanchez-Priego C, Valdes VJ, Saunders A, Ding J, Wu W-S, Clavel C, Wang J (2016) Zfp281 coordinates opposing functions of Tet1 and Tet2 in pluripotent states. Cell Stem Cell 19: 355-369. doi:10.1016/j.stem.2016.05.025

13. Tan L, Shi YG (2012) Tet family proteins and 5-hydroxymethylcytosine in development and disease. Development 139: 1895-1902. doi:10.1242/ dev.070771

14. Deplus R, Delatte B, Schwinn MK, Defrance M, Méndez J, Murphy N, Dawson MA, Volkmar M, Putmans P, Calonne E, et al (2013) TET2 and TET3 regulate GlcNAcylation and H3K4 methylation through OGT and SET1/ COMPASS. EMBO / 32: 645-655. doi:10.1038/emboj.2012.357

15. Vella P, Scelfo A, Jammula S, Chiacchiera F, Williams K, Cuomo A, Roberto A, Christensen J, Bonaldi T, Helin K, et al (2013) Tet proteins connect the $\mathrm{O}$-linked $\mathrm{N}$-acetylglucosamine transferase Ogt to chromatin in embryonic stem cells. Mol Cell 49: 645-656. doi:10.1016/ j.molcel.2012.12.019

16. Chen Q, Chen Y, Bian C, Fujiki R, Yu X (2013) TET2 promotes histone OGlcNAcylation during gene transcription. Nature 493: 561-564. doi:10.1038/nature11742

17. Williams K, Christensen J, Pedersen MT, Johansen JV, Cloos PAC, Rappsilber J, Helin K (2011) TET1 and hydroxymethylcytosine in transcription and DNA methylation fidelity. Nature 473: 343-348. doi:10.1038/nature10066

18. Ran FA, Hsu PD, Wright J, Agarwala V, Scott DA, Zhang F (2013) Genome engineering using the CRISPR-Cas9 system. Nat Protoc 8: 2281-2308. doi:10.1038/nprot.2013.143

19. Ying Q-L, Wray J, Nichols J, Batlle-Morera L, Doble B, Woodgett J, Cohen P, Smith A (2008) The ground state of embryonic stem cell self-renewal. Nature 453: 519-523. doi:10.1038/nature06968

20. Chambers I, Colby D, Robertson M, Nichols J, Lee S, Tweedie S, Smith A (2003) Functional expression cloning of Nanog, a pluripotency sustaining factor in embryonic stem cells. Cell 113: 643-655. doi:10.1016/ s0092-8674(03)00392-1

21. Chambers I, Silva J, Colby D, Nichols J, Nijmeijer B, Robertson M, Vrana J, Jones K, Grotewold L, Smith A (2007) Nanog safeguards pluripotency and mediates germline development. Nature 450: 1230-1234. doi:10.1038/ nature06403
22. Costa Y, Ding J, Theunissen TW, Faiola F, Hore TA, Shliaha PV, Fidalgo M, Saunders A, Lawrence M, Dietmann S, et al (2013) NANOG-dependent function of TET1 and TET2 in establishment of pluripotency. Nature 495: 370-374. doi:10.1038/nature11925

23. Xiong J, Zhang Z, Chen J, Huang H, Xu Y, Ding X, Zheng Y, Nishinakamura R, Xu G-L, Wang H, et al (2016) Cooperative action between SALL4A and TET proteins in stepwise oxidation of 5-methylcytosine. Mol Cell 64: 913-925. doi:10.1016/j.molcel.2016.10.013

24. Whyte WA, Orlando DA, Hnisz D, Abraham BJ, Lin CY, Kagey MH, Rahl PB, Lee TI, Young RA (2013) Master transcription factors and mediator establish super-enhancers at key cell identity genes. Cell 153: 307-319. doi:10.1016/j.cell.2013.03.035

25. Chambers I, Tomlinson SR (2009) The transcriptional foundation of pluripotency. Development 136: 2311-2322. doi:10.1242/dev.024398

26. Buecker C, Srinivasan R, Wu Z, Calo E, Acampora D, Faial T, Simeone A, Tan M, Swigut T, Wysocka J (2014) Reorganization of enhancer patterns in transition from naive to primed pluripotency. Cell Stem Cell 14: 838-853. doi:10.1016/j.stem.2014.04.003

27. Khoueiry R, Sohni A, Thienpont B, Luo X, Velde JV, Bartoccetti M, Boeckx B, Zwijsen A, Rao A, Lambrechts D, et al (2017) Lineage-specific functions of TET1 in the postimplantation mouse embryo. Nat Genet 49: 1061-1072. doi:10.1038/ng.3868

28. Hayashi K, Ohta H, Kurimoto K, Aramaki S, Saitou M (2011) Reconstitution of the mouse germ cell specification pathway in culture by pluripotent stem cells. Cell 146: 519-532. doi:10.1016/j.cell.2011.06.052

29. Guo G, Yang J, Nichols J, Hall JS, Eyres I, Mansfield W, Smith A (2009) Klf4 reverts developmentally programmed restriction of ground state pluripotency. Development 136: 1063-1069. doi:10.1242/dev.030957

30. Festuccia N, Halbritter F, Corsinotti A, Gagliardi A, Colby D, Tomlinson SR, Chambers I (2018) Esrrb extinction triggers dismantling of naïve pluripotency and marks commitment to differentiation. EMBO J 37: e95476. doi:10.15252/embj.201695476

31. Chazaud C, Yamanaka Y, Pawson T, Rossant J (2006) Early lineage segregation between epiblast and primitive endoderm in mouse blastocysts through the Grb2-MAPK pathway. Dev Cell 10: 615-624. doi:10.1016/j.devcel.2006.02.020

32. Festuccia N, Osorno R, Halbritter F, Karwacki-Neisius V, Navarro P, Colby D, Wong F, Yates A, Tomlinson SR, Chambers I (2012) Esrrb is a direct Nanog target gene that can substitute for Nanog function in pluripotent cells. Cell Stem Cell 11: 477-490. doi:10.1016/j.stem.2012.08.002

33. Rasmussen KD, Berest I, Keßler S, Nishimura K, Simón-Carrasco L, Vassiliou GS, Pedersen MT, Christensen J, Zaugg JB, Helin K (2019) TET2 binding to enhancers facilitates transcription factor recruitment in hematopoietic cells. Genome Res 29: 564-575. doi:10.1101/gr.239277.118

34. Wu H, D'Alessio AC, Ito S, Xia K, Wang Z, Cui K, Zhao K, Sun YE, Zhang Y (2011) Dual functions of Tet1 in transcriptional regulation in mouse embryonic stem cells. Nature 473: 389-393. doi:10.1038/nature09934

35. Hon GC, Song C-X, Du T, Jin F, Selvaraj S, Lee AY, Yen C, Ye Z, Mao S-Q, Wang B-A, et al (2014) 5mC oxidation by Tet2 modulates enhancer activity and timing of transcriptome reprogramming during differentiation. Mol Cell 56: 286-297. doi:10.1016/j.molcel.2014.08.026

36. Sardina JL, Collombet S, Tian TV, Gómez A, Di Stefano B, Berenguer C, Brumbaugh J, Stadhouders R, Segura-Morales C, Gut M, et al (2018) Transcription factors drive Tet2-mediated enhancer demethylation to reprogram cell fate. Cell Stem Cell 23: 727-741.e9. doi:10.1016/ j.stem.2018.08.016

37. Smith A (2017) Formative pluripotency: The executive phase in a developmental continuum. Development 144: 365-373. doi:10.1242/ dev.142679

38. Hooper M, Hardy K, Handyside A, Hunter S, Monk M (1987) HPRT-deficient (Lesch-Nyhan) mouse embryos derived from germline colonization by cultured cells. Nature 326: 292-295. doi:10.1038/326292a0 
39. Hayashi K, Saitou M (2013) Generation of eggs from mouse embryonic stem cells and induced pluripotent stem cells. Nat Protoc 8: 1513-1524. doi:10.1038/nprot.2013.090

40. Afgan E, Baker D, van den Beek M, Blankenberg D, Bouvier D, Čech M, Chilton J, Clements D, Coraor N, Eberhard C, et al (2016) The galaxy platform for accessible, reproducible and collaborative biomedical analyses: 2016 update. Nucleic Acids Res 44: W3-W10. doi:10.1093/nar/gkw343

41.. Babraham Bioinformatics. FastQC A Quality Control Tool for High Throughput Sequence Data. Available at https:// www.bioinformatics.babraham.ac.uk/projects/fastqc/. Accessed on April 26, 2010.

42. Langmead B, Salzberg SL (2012) Fast gapped-read alignment with Bowtie 2. Nat Methods 9: 357-359. doi:10.1038/nmeth.1923

43. Zhang Y, Liu T, Meyer CA, Eeckhoute J, Johnson DS, Bernstein BE, Nusbaum C, Myers RM, Brown M, Li W, et al (2008) Model-based analysis of ChIP-seq (MACS). Genome Biol 9: R137. doi:10.1186/gb2008-9-9-r137

44. Ramírez F, Ryan DP, Grüning B, Bhardwaj V, Kilpert F, Richter AS, Heyne S, Dündar F, Manke T (2016) deepTools2: A next generation web server for deep-sequencing data analysis. Nucleic Acids Res 44: W160-W165. doi:10.1093/nar/gkw257

45. Robinson JT, Thorvaldsdóttir H, Winckler W, Guttman M, Lander ES, Getz G, Mesirov JP (2011) Integrative genomics viewer. Nat Biotechol 29: 24-26. doi:10.1038/nbt.1754

cC) (i)

License: This article is available under a Creative Commons License (Attribution 4.0 International, as described at https://creativecommons.org/ licenses / by/4.0/). 